\title{
NECESIDAD DE UNA AUDITORÍA ACADÉMICA PARA LA UNIVERSIDAD NACIONAL MAYOR DE SAN MARCOS
}

\author{
THE NEED FOR AN ACADEMIC AUDIT FOR THE \\ NATIONAL UNIVERSITY OF SAN MARCOS \\ Yanetie Arm IDA Baca Morante* \\ Unidad de Posgrado de la Facultad de Ciencias Contables \\ Universidad Nacional Mayor de San Marcos - UNMSM / Lima - Perú \\ [Recepción: Agosto 2016/ Conformidad: Setiembre 2016]
}

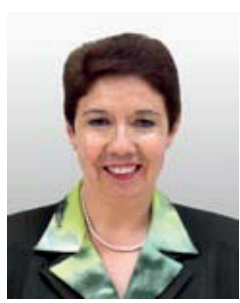

\section{RESUMEN}

El presente artículo "Necesidad de una auditoría académica para la Universidad Nacional Mayor de San Marcos", se basó en la investigación: "La importancia de la Auditoría Académica para la gestión de la Universidad Nacional Mayor de San Marcos en el siglo XXI 2012-2014". Efectuada para optar el grado de Doctor en Ciencias Contables y Empresariales. Los resultados manifestaron que existe un desfase de la Universidad Nacional Mayor de San Marcos, debido a las deficiencias en los procesos de su gestión; en especial, con respecto a la comunicación de sus integrantes sobre los planes y resultados de su ejecución, planteando la necesidad de una auditoría académica para la evaluación integral de sus procesos. El artículo se centra en los objetivos y las hipótesis concretadas, con un enfoque metodológico cuantitativo deductivo, utilizando el método estadístico probabilístico.

\section{Palabras Clave:}

Auditoría Académica; Universidad Nacional Mayor de San Marcos

\begin{abstract}
This article "The need for an academic audit for the National University of San Marcos" was based on research: "The importance of the Academic Audit for the management of the National University of San Marcos in the 21st century 2012-2014 ". Completed to obtain the degree of Doctor in Accounting and Business Sciences. The results showed that there is a lag of the Universidad Nacional Mayor de San Marcos, due to the deficiencies in the management processes. In particular, regarding the communication of its members on the plans and results of its execution, suggesting the need for an academic audit for the integral evaluation of its processes. The article focuses on the objectives and hypotheses, with a deductive quantitative methodological approach, using the probabilistic statistical method.
\end{abstract}

\section{Keywords:}

Academic Audit, Major National University of San Marcos

\footnotetext{
* Doctoranda en Políticas Públicas con mención en Defensa Nacional y Desarrollo Sotenible-UAP. Email: ybacam@unmsm.edu.pe
} 


\section{INTRODUCCIÓN}

La auditoría académica está diseñada especialmente para desarrollar la evaluación integral de instituciones educativas, en un ambiente de alta competitividad, siendo una herramienta básica para el proceso de evaluación: calidad y acreditación de las universidades e instituciones educativas en general. La implementación de la auditoría académica logra una evaluación de costo-beneficio, ahorro de tiempo y dinero, evitando riesgos en la gestión académica y gerencial. La auditoría académica se da con un equipo de profesionales multidisciplinarios e interdisciplinarios. Los resultados de esta herramienta da a los gestores, promotores y directivos en general, la base de información para resolver los problemas de los procesos de enseñanza-aprendizaje. Así se determina a la auditoría académica como el instrumento necesario para la reorganización de las universidades, para el logro de éxitos esperados.

Antes de plantear cambios en las universidades primero debe efectuarse una auditoría académica, para que las "comisiones reorganizadoras" cuenten previamente con un informe de auditoria académica, técnica y científicamente elaborada. La auditoría académica por desarrollarse en base a los principios de la auditoría en general, teniendo un campo de aplicación especializada en los procesos educativos, y por contar con procedimientos, técnicas e instrumentos e indicadores, enfocados a las actividades académicas, es una necesidad para el desarrollo de la educación en el Perú, en especial para las universidades. Los impactos esperados serian gestiones educativas de calidad, competitivas en condiciones de acreditación y certificación.

\section{EL PROBLEMA DE LA UNIVERSIDAD NACIONAL MAYOR DE SAN MARCOS}

La Universidad Nacional Mayor de San Marcos, fundada el 12 de mayo de 1551, como la primera universidad de América, cuenta en la actualidad con 62 carreras distribuidas en 20 facultades. La problemática moderna de la UNMSM se remonta al movimiento por la Reforma Universitaria de inicios de siglo XX, con sus principios, como los resume J.C. Mariátegui (1928) en la intervención de los alumnos en el gobierno de las universidades y de cátedra libre. Las diferentes leyes sobre la universidad han consignado en sus dispositivos los principios de la Reforma. La nueva Ley Universitaria 30220 del 8 de julio del 2014 presenta diferencias importantes, en relación a los principios de la Reforma Universitaria. En relación al principio de autonomía, con la creación de la SUNEDU - Superintendencia Nacional de Educación Superior Universitaria, adscrita al Ministerio de Educación, limita su autonomía al establecer una nueva relación entre el Estado y las universidades. En lo relativo al régimen de gobierno de las universidades, se da un avance cualitativo en relación a la Reforma Universitaria, la ley consagra la votación universal, personal, directa y ponderada por todos los docentes ordinarios y estudiantes matriculados para la elección del Rectory los Decanos correspondiendo $2 / 3$ a los docentes y $1 / 3$ a los estudiantes.

La problemática actual, en el siglo XXI, de la Universidad Nacional Mayor de San Marcos, se da en relación a su desfase ante los cambios de la sociedad en general. Este problema se constata al no aparecer en el ranking mundial de 400 universidades 2014-2015, publicado por la prestigiosa revista británica The Times Higher Education. La Universidad Nacional Mayor de San Marcos, por su historia y por su prestigio, para superar los problemas de su desarrollo, requiere ser evaluada por una auditoría académica para lograr un liderazgo educativo nacional e internacional.

\section{TEORÍAS SOBRE LOS CAMBIOS DE LA SO- CIEDAD MODERNA}

La investigación, "La importancia de la auditoría académica para la gestión de la Universidad Nacional Mayor de San Marcos en el siglo XXI 2012-2014”, para comprender el desfase de la Universidad Nacional Mayor de San Marcos, expone por marco teórico dos teorías relativas a la sociedad basada en el conocimiento y una teoría relativa a las organizaciones inteligentes en aprendizaje continuo.

La Teoría de las Olas de Cambio, expuesta por AlvinToffler (1981), interpreta los cambios de la sociedad en relación a la aceleración de la velocidad de los procesos. En su obra, "La Tercera Ola”, expone la revolución agrícola como la Primera Ola de Cambio radical que generó civilizaciones en toda la tierra, le 
sucede una Segunda Ola de cambio radical, basada en la revolución industrial. Lo importante en la teoría es el surgimiento, desde los años 60, de la Tercera Ola de cambio radical, de gran velocidad, con un núcleo basado en el conocimiento o la información, colisionando con la Segunda Ola, produciendo su caducidad y agonía. Para comprender la crisis y el desfase de las instituciones de la segunda Ola, el autor, devela los "códigos ocultos", que presiden el diseño de las estructuras de la Segunda Ola y la Tercera Ola. Siendo estos la Uniformización, Especialización, Sincronización, Concentración, Centralización, Maximización, para la Segunda Ola y la Diversificación, Sistematización, Flexibilización, Desconcentración, Descentralización, Minimización, para la Tercera Ola. Estos códigos son contradictorios, los de la Tercera Ola están generando la crisis de los códigos de la Segunda Ola, siendo necesario su aplicación para desarrollar las instituciones con el núcleo del conocimiento.

La teoría de la Sociedad Post Capitalista es una segunda visión general de la sociedad moderna, en base al conocimiento. Expuesta por Peter Drucker (1993), en el libro. "La Sociedad Post Capitalista", se descubre que la sociedad moderna ha dejado de ser capitalista, con el surgimiento de una nueva clase social, de cognitarios, que tienen en sus conocimientos su principal medio de producción. La nueva sociedad moderna lo integran organizaciones que compiten por captar a los especialistas, para lograr hacer productivo el conocimiento. La gestión moderna se encarga de la productividad del conocimiento.

La teoría de la Quinta Disciplina, es la visión que interpreta a las Organizaciones Inteligentes sistemáticas y en aprendizaje continuo, expuesta por Peter Senge (2005) y en su obra "La Quinta Disciplina", aplica la Teoría de Sistemas a las empresas y, a las organizaciones de la Sociedad. Expone a las organizaciones inteligentes que se basan en cinco disciplinas: 1. Pensamiento Sistémico, 2. Dominio Personal, 3. Modelos Mentales, 4. Visión Compartida. 5. Aprendizaje en Equipo. Estas cinco disciplinas se desarrollan como un conjunto, siendo el Pensamiento Sistémico, el elemento que integra a las demás "en un cuerpo coherente de teoría y práctica”.

\section{METODOLOGÍA}

La realización de la investigación sobre "La importancia de la auditoría académica para la gestión de la Universidad Nacional Mayor de San Marcos en el siglo XXI 2012 -2014”, se dio con un diseño metodológico cuantitativo deductivo, con un tipo de Investigación no experimental, siendo el alcance explicativo causal, y de clase transversal. Mediante un método de tratamiento de la información, estadístico probabilístico.

Por el método estadístico se determinó:

1) La Población de la Investigación: constituida por estudiantes y docentes de la UNMSM, cuya característica es formar parte de los procesos regulares de enseñanza y aprendizaje. Siendo la población de estudiantes (registrada en el Sistema Único de Matrícula enero-diciembre 2013) de 28,301. Y la población de los docentes regulares de 3,224.

2) Unidad de Análisis de la Población: Cada estudiante y docente de la UNMSM integrando los procesos de enseñanza y aprendizaje, constituyen las unidades de análisis de la población, de la investigación.

3) Población objetivo: Alumnos con matrícula regular de la Universidad Nacional Mayor de San Marcos y docentes regulares de la Universidad Nacional Mayor de San Marcos.

4) El diseño del tamaño de la muestra de la población de estudiantes y docentes UNMSM. El tamaño de la muestra se determinó considerando la estructura de la población objetivo del estudio, el cual se divide en dos sub poblaciones, ya mencionadas anteriormente. El esquema del muestreo, es el Muestreo Aleatorio Simple Estratificado, donde cada estrato es representativo a la población de alumnos y la población de docentes. Este diseño muestral permitirá que los resultados obtenidos de la encuesta sean extrapolables a la población objetivo.

5) Metodología de cálculo del tamaño de la muestra y su distribución: Para determinar el tamaño de muestra en cada estrato de estudio se determinó siguiendo la fórmula:

$$
n=\frac{\sum N_{h} p_{h} q_{h}}{N x d^{2}+\frac{\sum N_{h} p_{h} q_{h}}{N}}
$$


Para la distribución de la muestra en los estratos, se utilizó la distribución proporcional al tamaño de los estratos con la siguiente fórmula:

$$
n_{h=n}\left(\frac{N_{h}}{N}\right)
$$

El resultado determina el tamaño de la muestra de 375 para el estrato de estudiantes y de 43 para el estrato de docentes, dando una precisión de estudio del 95\% de confianza. La selección de los alumnos y docentes en la muestra es aleatoria, contemplando para ello, cuotas que se han establecido por facultad y Escuela Académica.

En la investigación, para transitar a la realidad empírica de la muestra, se construyeron instrumentos para la recolección de la información, efectuando correlaciones entre objetivos, variables e indicadores. También se elaboraron instrumentos de estructuras cerradas, dos cuestionarios de encuestas, uno destinada a los estudiantes, con preguntas cerradas y de escala. La segunda destinada a los docentes con preguntas igualmente cerradas y de escala. El análisis e interpretación de la información se realizó conforme el programa estadístico SPSS 21.

\section{RESULTADOS}

Los resultados de la investigación, develan las deficiencias y el desfase de la Universidad Nacional Mayor de San Marcos, y se interpretan en relación con los objetivos e hipótesis, en base a los cuadros estadísticos resultantes del procesamiento de datos.

\section{Los Objetivos:}

En el Objetivo General (O-G) se planteó identificar los problemas del desarrollo de la Universidad Nacional Mayor de San Marcos, relativos a la planificación, ejecución, monitoreo y evaluación de sus planes. Este objetivo es alcanzado por medio de los resultados de los tres Objetivos Específicos (O-E-1; O-E-2; O-E-3), planteados en la investigación, en relación a la problemática de la planificación, ejecución, monitoreo y evaluación de la gestión de la Universidad Nacional Mayor de San Marcos. La planificación, la ejecución de lo planeado, el monitoreo y la evaluación son importantes para una auditoría académica. Para ello, en la investigación se les preguntó a los estudiantes si conocían el plan anual de la universidad y de su facultad, los presupuestos de investigación, de los informes sobre la ejecución de los planes y si tienían conocimiento de sus evaluaciones. Asimismo, se preguntó a los docentes si participaban de la elaboración del plan de su facultad, del presupuesto de investigación aprobado, del informe de la ejecución del plan anual de su facultad y si tienen conocimiento de alguna auditoría realizada. Sin embargo, los resultados son abrumadoramente negativos, en relación a los estudiantes y en los docentes en menor medida. Como lo expresan las Tablas $\mathrm{N}^{\circ} 01,02$ y 03.

Tabla No 01

O-E 1 CONOCIMIENTO DE LOS ESTUDIANTES DEL PLAN ANUAL DE LA UNMSM Y DOCENTES PARTICIPANTES EN EL PLAN DE SU FACULTAD

\begin{tabular}{|c|c|c|c|c|c|c|}
\hline ESTRATO & $\begin{array}{c}\text { SI } \\
\text { MUESTRA }\end{array}$ & SI\% & $\begin{array}{c}\text { NO } \\
\text { MUESTRA }\end{array}$ & NO\% & $\begin{array}{c}\text { TOTAL MUES- } \\
\text { TRA }\end{array}$ & TOTAL \% \\
\hline ESTUDIANTES & 69 & 18.2 & 311 & 81.8 & 380 & 100 \\
\hline DOCENTES & 27 & 50.9 & 26 & 49.9 & 43 & 100 \\
\hline
\end{tabular}

Fuente: En base a la investigación: La importancia de la auditoría académica para la gestión de la Universidad Nacional Mayor de San Marcos 
Tabla No 02

O-E 2 CONOCIMIENTO DE LOS ESTUDIANTES DEL INFORME DE EJECUCIÓN DEL PLAN ANUAL DE LA UNMSM Y DOCENTES DELINFORME DE LA EJECUCIÓN DEL PLAN DE SU FACULTAD

\begin{tabular}{|c|c|c|c|c|c|c|}
\hline ESTRATO & $\begin{array}{c}\text { SI } \\
\text { MUESTRA }\end{array}$ & SI\% & $\begin{array}{c}\text { NO } \\
\text { MUESTRA }\end{array}$ & NO\% & $\begin{array}{c}\text { TOTAL MUES- } \\
\text { TRA }\end{array}$ & TOTAL \% \\
\hline ESTUDIANTES & 20 & 5.3 & 360 & 94.7 & 380 & 100 \\
\hline DOCENTES & 24 & 45.3 & 29 & 54.7 & 43 & 100 \\
\hline
\end{tabular}

Fuente: En base a la investigación: La importancia de la auditoría académica para la gestión de la Universidad Nacional Mayor de San Marcos

Tabla No 03

O-E 3 CONOCIMIENTO DE LOS ESTUDIANTES DEL MONITOREO Y EVALUACIÓN DE LA PLANIFICACIÓN DE UNMSM Y DOCENTES CONOCIMIENTO DE LA REALIZACIÓN DE ALGUNA AUDITORÍA ACADÉMICA

\begin{tabular}{|c|c|c|c|c|c|c|}
\hline ESTRATO & $\begin{array}{c}\text { SI } \\
\text { MUESTRA }\end{array}$ & SI\% & $\begin{array}{c}\text { NO } \\
\text { MUESTRA }\end{array}$ & NO\% & $\begin{array}{c}\text { TOTAL } \\
\text { MUESTRA }\end{array}$ & $\begin{array}{c}\text { TOTAL } \\
\%\end{array}$ \\
\hline ESTUDIANTES & 53 & 13 & 327 & 86.1 & 380 & 100 \\
\hline DOCENTES & 24 & 45.3 & 29 & 54.7 & 43 & 100 \\
\hline
\end{tabular}

Fuente: En base a la investigación: La importancia de la auditoría académica para la gestión de la Universidad Nacional Mayor de San Marcos

Los resultados de los objetivos expuestos manifiestan el desconocimiento de la mayoría de estudiantes y de docentes de la planificación, ejecución y evaluaciones de la gestión de la UNMSM. Se interpretan como manifestación de las deficiencias en la gestión, y del desfase de la Universidad, respecto de las organizaciones modernas sistémicas, basadas en la productividad del conocimiento, en especial de las Organizaciones Inteligentes. Los resultados en relación a la gestión de la UNMSM, manifiestan que no es sistémica, carece de una visión compartida, no se da el trabajo en equipo y determinan la necesidad de su reconversión. Además, los resultados deben considerarse como tendencias contrarias al desarrollo de la UNMSM, en base a la productividad del conocimiento que son ratificados en la comprobación de las hipótesis.

\section{Las hipótesis:}

En la investigación "La importancia de la Auditoría Académica para la gestión de la Universidad
Nacional Mayor de San Marcos en el siglo XXI 20122014", se plantearon cuatro hipótesis, una general y tres específicas.

La Hipótesis General (H-G)afirmó que la UNMSM tiene deficiencias en la planificación, ejecución y evaluación por su desfase y no adecuación a los códigos de la Tercera Ola de la revolución del conocimiento. Reformulado en una estructura causal de las variables; La Tercera Ola de la revolución del conocimiento $(\mathrm{X})$, por el desfase en relación a estos cambios $(\mathrm{Z} 1)$ y no adecuación a los códigos de estas Revoluciones (Z2) producen en la UNMSM el atraso en su desarrollo relativo a su planificación, al monitoreo y a la evaluación de lo planificado (Y1) generando desconcierto en la Universidad (Y2). Se comprueba la hipótesis, contrastando sus variables, por medio de sus indicadores, con la realidad empírica. En el presente artículo, se resume esta contrastación de la Hipótesis General (H-G) en la Tabla N ${ }^{\circ} 04$. 
Tabla No 04

COMPROBACIÓN DE LAS VARIABLES DE LA H-G

\begin{tabular}{|c|l|c|c|c|c|c|c|}
\hline ESTRATO & INDICADOR VARIABLE & $\begin{array}{c}\text { SI } \\
\text { MUESTA }\end{array}$ & SI \% & $\begin{array}{c}\text { NO } \\
\text { MUESTRA }\end{array}$ & NO \% & $\begin{array}{c}\text { TOTAL } \\
\text { MUESTRA }\end{array}$ & $\begin{array}{c}\text { TOTAL } \\
\%\end{array}$ \\
\hline ESTUDIANTE & (X) Computadoras en aula & 44 & 11.5 & 336 & 88.4 & 380 & 100 \\
\hline ESTUDIANTE & (Z) Forman Círculos Aula & 74 & 19.5 & 306 & 80.5 & 380 & 100 \\
\hline ESTUDIANTE & $\begin{array}{l}\text { (Z) Aprender a investigar } \\
\text { desde el inicio de la carrera }\end{array}$ & 288 & 76.6 & 92 & 23.4 & 380 & 100 \\
\hline ESTUDIANTE & (Y) Grupo de Investigación & 16 & 4.2 & 364 & 95.8 & 380 & 100 \\
\hline DOCENTES & (X) Internet en aula & 34 & 64 & 19 & 35.8 & 43 & 100 \\
\hline DOCENTES & (Z) Artículos científicos & 18 & 47.2 & 25 & 52.8 & 43 & 100 \\
\hline DOCENTES & (Y) Proyectos de Invest & 15 & 26.3 & 38 & 71.7 & 43 & 100 \\
\hline
\end{tabular}

Fuente: En base a la investigación: La importancia de la auditoría académica para la gestión de la Universidad Nacional Mayor de San Marcos

Los resultados de la comprobación de la Hipótesis General (H-G), manifiestan que la mayoría de estudiantes no se forman para la sociedad moderna diseñada con los códigos de la Tercer Ola y que requiere de investigadores, también que la mayoría de docentes no están formando a los estudiantes para esta sociedad. Se interpretan estos resultados como manifestación de las deficiencias en el liderazgo de su gestión, de la planificación, ejecución y evaluación, dado a que la UNMSM está desfasada respecto a la Tercera Ola de ambio, y de las organizaciones modernas participativas, sistémicas, que hacen productivo el conocimiento. Este resultado evalúa a la
Universidad en relación a los cambios innovadores que se dan en la sociedad.

La Hipótesis Específica 1 (H-E-1). Formula que: La Universidad adolece de deficiencias en la Planificación Anual de su Dirección y de Gobierno, por no contar con órganos especializados de apoyo a esta actividad. Reformulada en una estructura causal de sus variables. El funcionamiento de las estructuras de Dirección y Gobierno de la UNMSM $(\mathrm{X})$ producen, al no contar con órganos de apoyo especializados $(Z)$, deficiencias en la planificación anual de la universidad (Y). (Ver Tabla $\mathrm{N}^{\circ} 05$ )

Tabla No 05

COMPROBACIÓN DE LAS VARIABLES DE LA H-E-1

\begin{tabular}{|c|c|c|c|c|c|c|c|}
\hline \multirow{2}{*}{ ESTRATO } & INDICADOR VARIABLE & $\begin{array}{c}\text { SI } \\
\text { MUESTA }\end{array}$ & SI \% & $\begin{array}{c}\text { NO } \\
\text { MUESTRA }\end{array}$ & NO \% & $\begin{array}{c}\text { TOTAL } \\
\text { MUES- } \\
\text { TRA }\end{array}$ & $\begin{array}{c}\text { TOTAL } \\
\%\end{array}$ \\
\hline $\begin{array}{c}\text { ESTUDIAN- } \\
\text { TE }\end{array}$ & $\begin{array}{c}\text { (X) Conoce presupuesto de inves- } \\
\text { tigación }\end{array}$ & 15 & 3.9 & 365 & 96.1 & 380 & 100 \\
\hline $\begin{array}{c}\text { ESTUDIAN- } \\
\text { TE }\end{array}$ & (Z) Decano informa plan facultad & 94 & 24.7 & 286 & 75.3 & 380 & 100 \\
\hline $\begin{array}{c}\text { ESTUDIAN- } \\
\text { TE }\end{array}$ & (Y) Ha realizado publicaciones & 16 & 4.2 & 364 & 95.8 & 380 & 100 \\
\hline $\begin{array}{c}\text { DOCENTE } \\
\text { (X) Conoce presupuesto de su } \\
\text { facultad }\end{array}$ & 13 & 28.3 & 30 & 71.7 & 43 & 100 \\
\hline DOCENTE & $\begin{array}{c}\text { (Z) Participa en el Plan Anual de } \\
\text { su Facultad }\end{array}$ & 27 & 50.9 & 26 & 49.1 & 43 & 100 \\
\hline DOCENTE & (Y) Bonificación por Investigación & 20 & 37.7 & 23 & 53.3 & 43 & 100 \\
\hline
\end{tabular}

Fuente: En base a la investigación: La importancia de la auditoría académica para la gestión de la Universidad Nacional Mayor de San Marcos

60/ QVIPURAMAYOC | Vol. 24(46) 2016 
Los resultados de la comprobación de la Hipótesis Específica 1(H-E-1), develan que la mayoría de estudiantes no conoce los planes ni los presupuestos de la Universidad y de sus Facultades. Asimismo, una mayoría de docentes no participa en la elaboración de los planes de su facultad. Estos resultados comprueban las deficiencias de los órganos de gobierno para la planificación de la UNMSM y que no cuentan con especialistas en las metodologías de la planificación moderna, que apoyen en la proyección de organizaciones modernas participativas, que hagan productivo el conocimiento. De ahí, las deficiencias en la planificación de la UNMSM.

La Hipótesis Específica 2 (H-E-2). Plantea: La UNMSM presenta deficiencias en la Ejecución de sus Planes Anuales, por su desfase de sus velocidades en relación al cambio y por no contar con una metodología eficaz que permita hacer su seguimiento. Se reformuló la estructura causal de sus variables: Por no contar con una metodología eficaz y eficiente de monitoreo (X1) y de evaluación (X2), la UNMSM presenta deficiencias en el monitoreo y evaluación de su plan anual (Y). (Ver Tabla $\left.\mathrm{N}^{\circ} 06\right)$

Tabla No 06

COMPROBACIÓN DE LAS VARIABLES DE LA H-E-2

\begin{tabular}{|c|c|c|c|c|c|c|c|}
\hline ESTRATO & INDICADOR VARIABLE & $\begin{array}{c}\text { SI } \\
\text { MUESTA }\end{array}$ & SI \% & $\begin{array}{c}\text { NO } \\
\text { MUESTRA }\end{array}$ & NO $\%$ & $\begin{array}{l}\text { TOTAL } \\
\text { MUESTRA }\end{array}$ & $\underset{\%}{\text { TOTAL }}$ \\
\hline ESTUDIANTE & $\begin{array}{l}\text { (X) Conoce Informe Anual del } \\
\text { Rector }\end{array}$ & 20 & 5.3 & 360 & 94.7 & 380 & 100 \\
\hline ESTUDIANTE & $\begin{array}{l}\text { (Z) Conoce Informe Anual del } \\
\text { Decano }\end{array}$ & 42 & 11.1 & 338 & 88.9 & 380 & 100 \\
\hline ESTUDIANTE & $(\mathrm{Y})$ & & & & & 360 & 100 \\
\hline DOCENTE & $\begin{array}{l}\text { (X) Conoce presupuesto Ejecutado } \\
\text { InvestFac. }\end{array}$ & 8 & 15.1 & 35 & 84.9 & 43 & 100 \\
\hline DOCENTE & $\begin{array}{l}\text { (X) Conoce Ejecución Plan Anual } \\
\text { Facultad }\end{array}$ & 14 & 45.3 & 29 & 54.7 & 43 & 100 \\
\hline DOCENTE & $\begin{array}{l}\text { (Y) Artículos científicos para la } \\
\text { Facultad }\end{array}$ & 18 & 47.2 & 25 & 52.8 & 43 & 100 \\
\hline
\end{tabular}

Fuente: En base a la investigación: La importancia de la auditoría académica para la gestión de la Universidad Nacional Mayor de San Marcos

Los resultados en relación a la comprobación de la Hipótesis Específica 2 (H-E-2), devela el desconocimiento de la mayoría de estudiantes del monitoreo y la evaluación de la ejecución de planes ni los presupuestos de la Universidad y de sus Facultades, asimismo, la mayoría de docentes desconoce sobre la ejecución de los planes de su facultad, Estos resultados comprueban que UNMSM no cuenta con una metodología eficaz de monitoreo y evaluación, que determinen las deficiencias en la ejecución de los planes de la UNMSM, y analicen las deficiencias de los procesos de investigación y de aprendizaje.
La Hipótesis Específica 3 (H-E-3), conjetura que: La UNMSM adolece de deficiencias en la evaluación de la ejecución de sus Planes Anuales, por no contar con Auditorías Académicas modernas y por carecer de una metodología eficaz para la evaluación respectiva. Se reformula en su estructura de variables: Por no contar con una metodología eficaz y eficiente de evaluación $(X)$, por no contar con una Auditoría Académica moderna (Z), la UNMSM presenta deficiencias en la evaluación de su plan anual (Y).

(Ver Tabla N 07 en la siguiente página) 
Tabla No 07

COMPROBACIÓN DE LAS VARIABLES DE LA H-E-3

\begin{tabular}{|c|c|c|c|c|c|c|c|}
\hline ESTRATO & INDICADOR VARIABLE & SI & SI $\%$ & $\begin{array}{c}\text { NO } \\
\text { MUESTRA }\end{array}$ & NO \% & $\begin{array}{c}\text { TOTAL } \\
\text { MUES- } \\
\text { TRA }\end{array}$ & $\begin{array}{c}\text { TOTAL } \\
\%\end{array}$ \\
\hline $\begin{array}{c}\text { ESTUDIAN- } \\
\text { TE }\end{array}$ & $\begin{array}{c}\text { (X) Conocimiento de Evaluación } \\
\text { UNMSM }\end{array}$ & 53 & 13.9 & 327 & 86.1 & 380 & 100 \\
\hline $\begin{array}{c}\text { ESTUDIAN- } \\
\text { TE }\end{array}$ & $\begin{array}{c}\text { (Z) Conocimiento Informe Ejecu- } \\
\text { ción Plan de Univ. }\end{array}$ & 20 & 5.3 & 360 & 94.7 & 380 & 100 \\
\hline $\begin{array}{c}\text { ESTUDIAN- } \\
\text { TE }\end{array}$ & $\begin{array}{c}\text { (Y) Información sobre Becas } \\
\text { DOCENTE }\end{array}$ & $\begin{array}{c}\text { (X) Conocimiento de ejecución } \\
\text { del Plan Anual de su Facultad. }\end{array}$ & 14 & 45.3 & 29 & 54.7 & 43 \\
\hline DOCENTE & $\begin{array}{c}\text { (Z) Conocimiento de una Audito- } \\
\text { ría Acad. a la Univ }\end{array}$ & 14 & 45.3 & 29 & 54.7 & 43 & 100 \\
\hline DOCENTE & $\begin{array}{c}\text { (Y) Presentación de Proyectos a } \\
\text { concursos Internacionales }\end{array}$ & 15 & 28.3 & 28 & 71.7 & 43 & 100 \\
\hline
\end{tabular}

Fuente: En base a la investigación: La importancia de la auditoría académica para la gestión de la Universidad Nacional Mayor de San Marcos

Los resultados en relación a la comprobación de la Hipótesis Específica 3 (H-E-3), expone el desconocimiento de los estudiantes sobre la evaluación del plan anual de UNMSMy de su ejecución. La mayoría de docentes desconoce la necesidad de la ejecución del plan anual de su facultad y de una auditoría académica para la Universidad. Estos resultados explican porque una mayoría de estudiantes no conocen de los concursos de becas de estudios y una mayoría de docentes desconoce sobre los concursos internacionales de investigación. Además, los resultados descubren deficiencias en la evaluación del Plan Anual de la UNMSM y la necesidad de evaluarla mediante una Auditoría Académica, por ser especializada para las instituciones educativas.

\section{CONCLUSIONES}

De los resultados obtenidos en la investigación. La importancia de la auditoría Académica para la gestión de la Universidad Nacional Mayor de San Marcos en el siglo XXI 2012-2014; tanto de los objetivos y de la comprobación de sus Hipótesis, se deducen las siguientes conclusiones:

En relación a los objetivos, teniendo como referente a las Organizaciones Inteligentes, de aprendizaje continuo, los resultados obtenidos, permiten concluir que la UNMSM adolece de pensamiento sistemático. Su población no es participativa porque carece de una visión de futuro compartido, no domina el trabajo en equipo, no se incentiva los modelos mentales y el dominio personal para su desarrollo. Los desconocimientos captados sobre la planificación, ejecución y evaluación de la gestión, conducen a esta conclusión, siendo necesario planificar una innovación de la Universidad, en la base a la aplicación de una auditoría académica.

En relación a las Hipótesis y teniendo en cuenta su relación con los objetivos planteados, se puede concluir, que los resultados comprobaron la Hipótesis General, determinando la existencia de un desfase de la UNMSM respecto a la Tercera Ola de cambio, por no estar desarrollando el conocimiento con miras a su producción y productividad, siendo necesaria su adecuación. Asimismo, en las Hipótesis Espercíficas se confirma los objetivos alcanzados, explicando los efectos de las deficiencias de la planificación, ejecución, monitoreo, y evaluación de la gestión de la UNMSM.

Concluyéndose en la investigación la urgente necesidad de aplicar a la UNMSM, una auditoría académica, para proyectar sudesarrollo e innovación en relación a la sociedad moderna del conocimiento. 


\section{REFERENCIAS BIBLIOGRÁFICAS}

1. TOFFLER.A. (1981) La Tercera Ola, Bogotá Plaza \& Janes.

2. TOFFLER. A (1994), El cambio del Poder, 2da. Edición, Barcelona, Plaza \&Janes.

3. DRUCKER P. (2003) La Sociedad Post Capitalista, Séptima Reimpresión, Colombia Editorial Sudamericana.

4. SENGE, P. (2005) La Quinta Disciplina, Argentina. Ediciones Granica

5. MARIATEGUi J.C. (2003) Temas de Educación. Décima sexta edición. Perú Empresa Editora Amauta.
6. MARIATEGUI (2007) 7 Ensayos de Interpretación de la realidad peruana. Septuagésima cuarta edición. Perú. Viuda de Mariátegui e Hijos S.A. Librería Editorial Miraflores, Lima

7. EGUSQUIZA C. (2004) Auditoría Académica. Artículo publicado en la Revista Quipukamayoc. Facultad de Ciencias Contables UNMSM. Lima

8. THE TIMES HIGHER EDUCATION (2015) Universidad Ranking Mundial (2014-2015), Disponible en: https://www. timeshighereducation.co,uk/world-university-rankings/2015/world-ramking (2015,9 de agosto) 